\title{
MICROSCOPIC SPECTRAL IMAGING ANALYSIS OF CRYSTALLINE POLYCYCLIC AROMATIC HYDROCARBONS
}

\author{
Chanan Sluszny, Vladimir V. Gridin, Valery Bulatov \\ and Israel Schechter \\ Department of Chemistry and \\ Institute of Catalysis Science and Technology, \\ Technion-Israel Institute of Technology, \\ Haifa 32000, Israel
}

\begin{abstract}
A new spectral imaging approach, based on an imaging microscope coupled to a Fourier transform spectrometer, was applied for direct analysis of polycyclic aromatic hydrocarbons (PAHs) deposited onto solid matrices. Single crystals of several PAHs were grown and their fluorescence spectra were analyzed. The fluorescence characteristics, related to analytical reliable quantification of solid PAH compounds, were investigated. In particular, we have addressed the following parameters: (a) crystal-alignment effects upon the read-outs of the microscopic imaging set-up; (b) effects associated with either the crystal edge or the crystal surface originated fluorescence spectra; (c) inter-crystal self-absorption related processes. The understanding of these effects is important for a proper sampling of the PAHs and is essential for obtaining reliable quantification.
\end{abstract}

\section{INTRODUCTION}

Polycyclic Aromatic Hydrocarbons (PAHs) are known as a family of potentially carcinogenic/mutagenic compounds $/ 1,2 /$. These substances are produced in a variety of industrial processes and are usually associated with incomplete combustion of fossil fuels. Due to their relatively low volatility and high toxicity /3/ PAHs are considered as hazardous substances. Thus, routine analysis and direct monitoring of PAH depositions are of utmost 
importance.

On account of their high molecular weight and concomitant low vapor pressure, PAH compounds have been considered to be associated exclusively with particulate matter $/ 4,5 /$. Larger fractions of particulate contaminants would precipitate within a few days $/ 4 /$, thus, polluted soil and water are frequently found near industrial facilities.

Conventional analysis of PAHs performed on environmental samples is usually based on laboratory procedures performed upon the field-collected samples $/ 6-8 /$. These methods, usually based on extraction followed by liquid or gas chromatographic analysis provide good analytical results, however, quite often are time consuming. The latter may also result in unintentional sample contamination.

In addition to the laboratory separation methods, several optical principles of analysis were suggested for fast and on-site analysis of PAHs. The most promising ones are based on the characteristic fluorescence of these materials 19/. Several applications of lasers and time-resolved techniques for on-line monitoring of PAHs in groundwater have recently been developed /9/. Analysis of PAHs in soils, however, is a much more difficult task, since one must first extract the pollutants from the soil samples. The analysis of these extracts by fluorescence is well established, but direct measurements of these pollutants in soil have not yet been developed $/ 10,11 /$.

In a recent study /12/ we proposed a fluorescence imaging apparatus for analysis of PAH depositions. The method was based on direct analysis of the UV induced fluorescence, collected by a microscope array, which was coupled to a Fourier transform imaging spectrometer. This way, a full fluorescence spectrum was calculated at each pixel of the image produced on a built-in CCD camera. It was demonstrated that Fourier Transform Imaging Microscopy (FTIM) can be applied for analysis of PAH particulates deposited on soil specimens. Appropriate calibration curves suggest absolute detection limits of a few $\mathrm{pg}$ for perylene and pyrene.

However, it has been concluded that reliable quantification of $\overline{\mathrm{PAH}}$ particulates requires further investigation of several features inherent to the microscopic imaging analysis itself. In this report we discuss these findings in regard to FTIM studies of crystalline PAH compounds. Significance of the data in respect of quantification algorithms for monitoring particulate $\mathrm{PAHs}$ is emphasized. 


\section{EXPERIMENTAL}

\section{Sample preparation:}

Perylene (Fluka, 99\%) and anthracene (Fluka, 99\%), were used without further purification. Crystalline PAHs were grown from saturated solutions of hexane, toluene and benzene. After the solvents had evaporated, perylene rectangular crystals were obtained from hexane and toluene solutions, while perylene tabular-rectangular crystals were obtained from benzene solution. Tabular-rectangular anthracene crystals were also obtained from hexane solutions. The crystals were placed on a flat surface for imaging analysis.

\section{Experimental setup:}

The spectral imaging experimental setup, schematically shown in Fig. 1, is similar to the one previously reported $/ 12 \%$. It is composed of a fluorescence microscope (Axiolab, Carl Zeiss, Germany), a Fourier transform imaging spectrometer, a cooled CCD detector and a PC coupled data acquisition system. The excitation light $(365 \mathrm{~nm})$ was transferred through the microscope optics and objective such that it reached the sample exactly at the focal plane. The back emitted fluorescence passed through the same optics towards the spectrometer, where a special optical filter blocked the reflected UV light (below $390 \mathrm{~nm}$ ). The microscope scene was passed, as an image, to a Sagnac type Fourier transform spectrometer, mounted on the microscope. The spectrometer was equipped with imaging lenses, such that the full image from the microscope could be processed simultaneously. (FIPA 2000, GreenVision, Ltd., Israel)

The obtained interferograms were recorded by an electrothermally cooled, 12 bit, CCD camera (Hamamatsu, 4880-81, Japan), covering up to $480 \times 640$ pixels. The CCD pixel size was $9.9 \times 9.9 \mu \mathrm{m}$, which corresponds to about $\mathrm{I} \times \mathrm{I}$ $\mu \mathrm{m}$ at the object plane, when using a $\times 10$ microscope objective. The imaging interferograms thus obtained were transferred to a PC for Fourier transforming, resulting in full fluorescence spectrum at each $C C D$ pixel.

\section{Data analysis:}

The data analysis program was similar to the one previously reported $/ 12 /$. First, the fluorescence of each PAH particulate emitter was detected and separated from the background radiation. Speciation was based on 


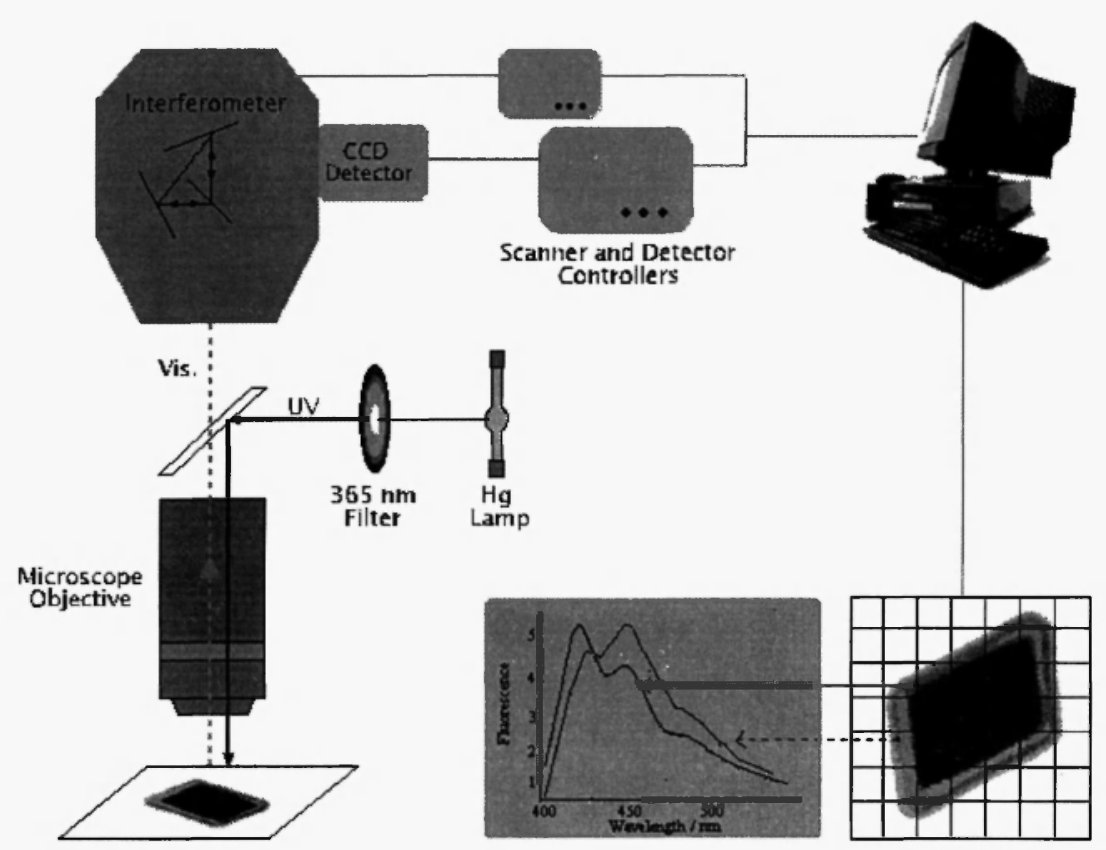

Fig. 1: Experimental setup for microscopic imaging fluorescence analysis.

comparison to reference spectra and quantification was based on the so-called luminescence volume, which is an integral of the luminescence intensity at a given spectral range, over the area of the relevant light emitter $/ 12 \%$. In the following, these are referred as "fluorescence intensities". In the current study, we acquired such information for each pixel, such that detailed spectral and spatial information was available for single particulate emitters, at a resolution of ca. $1 \mu \mathrm{m}$.

\section{Procedures:}

Photoluminescence analysis was performed on a number of microcrystals, whose radiative areas were determined at a resolution of $1 \mu^{2}$. Specified regions of the crystal were analyzed independently. For the investigation of the crystal alignment effects, a perylene crystal was deposited on a ca. $100 \mu \mathrm{m}$ glass wire. The latter was rotated around its long axis, exposing several orientations of the crystal to the microscope objective. 
Many PAH crystals were examined; however, only representative images are shown here.

\section{SEM analysis:}

Scanning electron microscopy was applied to perylene crystals in order to provide independent information on crystal morphology. The crystals were placed on a flat surface, gold coated and scanned with a scanning electron microscope (GEOL, GSM 5400).

\section{RESULTS AND DISCUSSION}

Several microscopic imaging characteristics of crystalline PAH compounds were investigated. These include the light wave-guiding properties, physical dimensions, self-absorption, sample alignment (with respect to the incoming excitation light) and structural defects. Two major routes of data presentation are followed: the fluorescence intensity data and spectral features obtained by FTIM analysis of PAH particulates.

\section{Fluorescence intensity data of FTIM}

The FTIM intensity data obtained from the crystalline PAH compounds requires careful consideration. Peculiar intensity features occur whenever some of the specimen's dimensions are considerably larger (say, 10 times or so) than the wavelength of the UV-excitation used. A rather straightforward mass quantification of PAH particulates by FTIM becomes then ambiguous. In what follows we point out several, most frequent, observations met in such cases.

a. Fluorescence intensity feature due to wave-guiding effects.

In Fig. $2 \mathrm{a}$ we present a scanning electron microscope image of a typical perylene micro-crystal, grown from perylene/hexane solution. The crystal has a rectangular flat structure and appears to be of a uniform thickness.

The corresponding 3D-plot of the wavelength-integrated fluorescence intensity provided by FTIM is shown in Fig. 2b. One observes that intensive fluorescence is emitted near the crystal edges and it is substantially smaller 

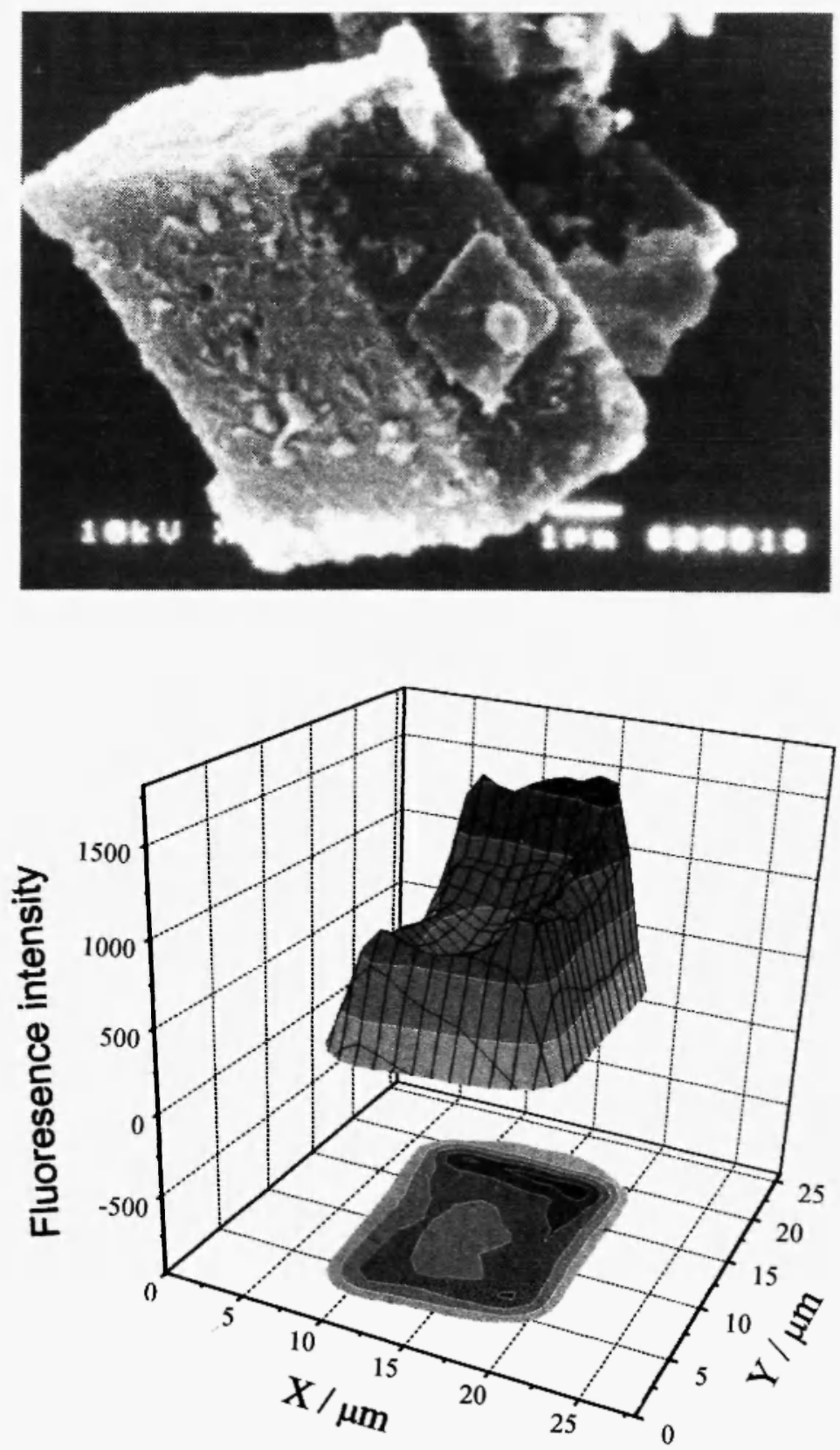

Fig. 2: (a) Scanning Electron Microscope image of a perylene crystal. (b) Corresponding 3D-plot of the wavelength-integrated fluorescence intensity. 
otherwise. On the grounds of a uniform sample thickness any simple geometrical interpretation of intensity variations, as in Fig. $2 b$, should fail.

On the other hand, multiple internal reflections of randomly emitted photons would produce enhanced fluorescence at the narrow crystal facets $113-15 /$. In other words, the wave-guiding interpretation of this data seems plausible. Similar results were also obtained for the anthracene crystals studied.

It should be noted, however, that within each batch of as-grown crystals there always were some poorly fluorescing ones. When investigated by the microscope imaging facility, these crystals appeared to lack the aforementioned wave-guiding effect. If the thickness of such crystals were of the order of (or less than) the fluorescence wavelength itself, the waveguiding of the emitted light would not take place. Alternatively, though, quite low emission yields found there could be due to the surface quenched fluorescence. This is known to be promoted by such structural imperfections as various bulk, surface or point defects $/ 16 /$.

b. Fluorescence intensity as a function of crystal area.

Many crystal batches were analyzed, resulting in calibration plots shown in Fig. 3. The linear regression fits indicate a reasonable correspondence between the fluorescence intensity of crystal and its physical size.

The large data spread of Fig. 3 is attributed to morphology related effects. In particular, the fluorescence emitted by the particulate material could be related to surface defects $/ 16 /$. Moreover, the emitted light mostly originates from a surface layer rather than from the crystal's bulk. This is supported by our preliminary experiments in which an extinction coefficient of ca. $10^{3}$ was measured from a solid layer of these substances. This, in turn, leads to a substantial absorbtion of the excitation light, resulting in a light penetration depth of about $1-2 \mu \mathrm{m}$ only.

In other words, the fluorescence based microscopic imaging turns out to be a surface layer analytical probe. As such, the method picks up the apparent area of the crystals only. Such an area is the one which is determined at the plane facing the microscope objective.

This point is best illustrated by Fig. 4. This figure shows the fluorescence intensity contour maps of a single perylene crystal, positioned at different orientations towards the microscope objective. The imaged size of the crystal changes from $660 \mu \mathrm{m}^{2}$ to $1130 \mu \mathrm{m}^{2}$ on account of its orientation with respect to the microscope's objective. It should be noted, however, that the 


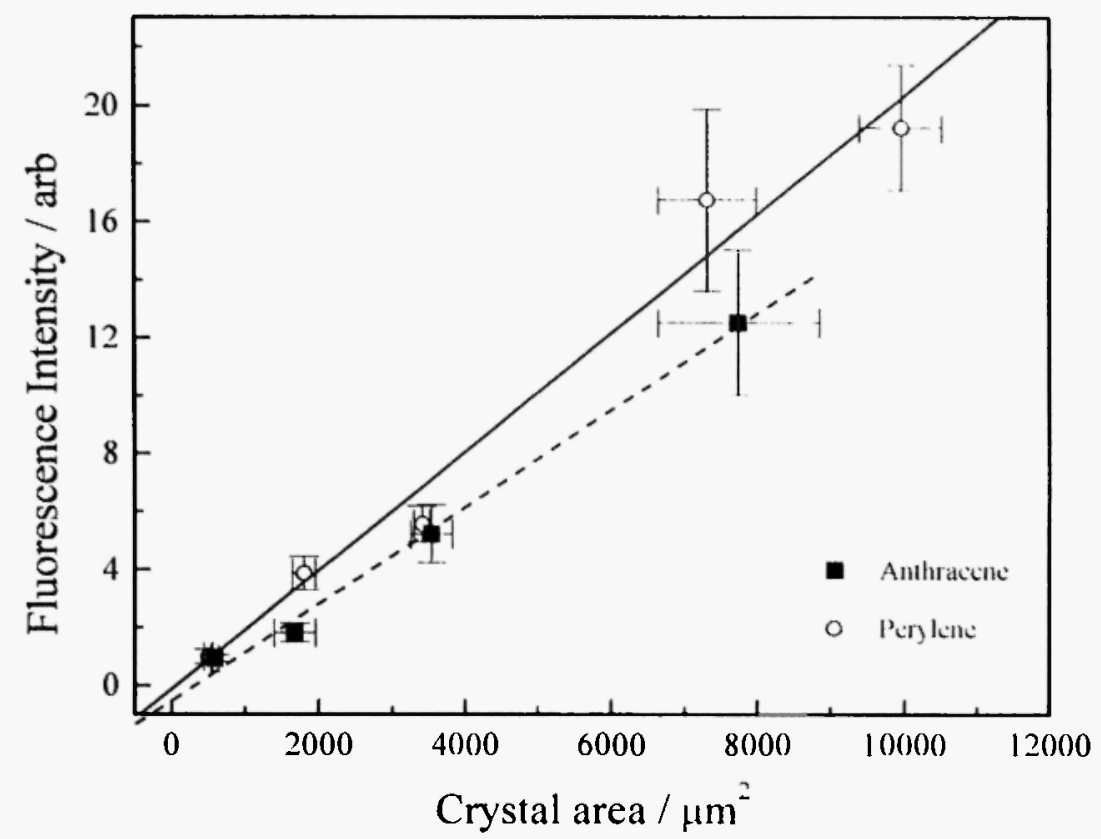

Fig. 3: Calibration plots for the fluorescence intensity as a function of the size of perylene and anthracene crystals.

fluorescence intensity related to the different crystal orientations agrees well with the imaged size.

These results, which have indicated a thin layer originated fluorescence, must be considered for quantification purposes. That is, because only a suriace layer is actually being probed, a large mass of the particulate matter may not be accounted for. This problem should be resolved by an appropriate correction algorithm, which takes into account the actual distribution of morphological characteristics of the crystallites.

An additional quantification problem that requires a proper correction is related to the fluorescence intensity variations measured at the various regions of any particular crystal. Recalling the data of Fig. $2 \mathrm{~b}$ we note that for perylene, on average, the crystal-edge related fluorescence was about 5 times higher than that obtained from the crystal's central region. This effect was somewhat smaller for anthracene crystals, whereby a three-fold intensity increase was observed. 


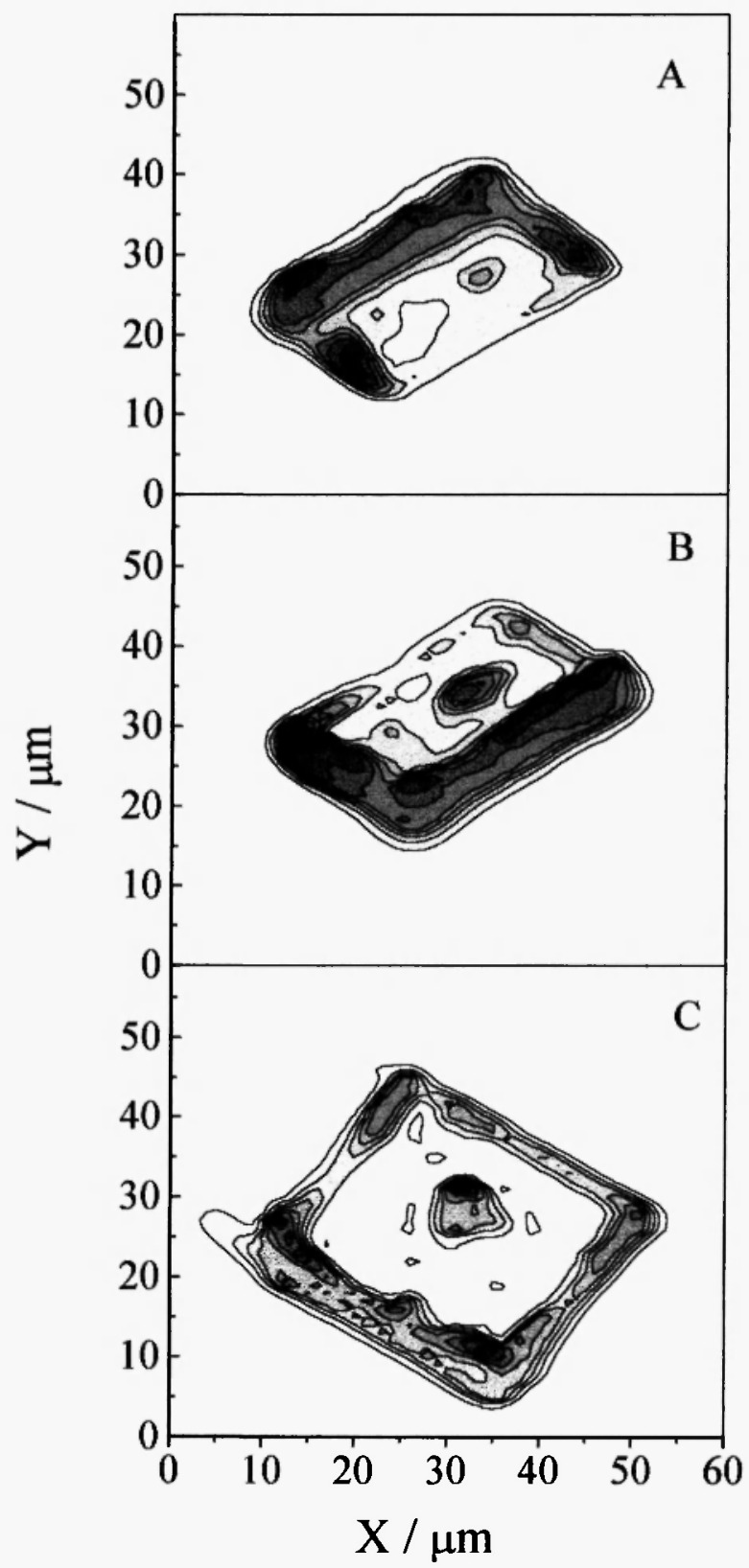

Fig. 4: Fluorescence intensity contour maps of a single perylene crystal aligned at different orientations towards the microscope objective. The area attributed to the imaged crystal was: $A: 660 \mu \mathrm{m}^{2}, \mathrm{~B}: 720$ $\mu \mathrm{m}^{2}, \mathrm{C}: 1130 \mu \mathrm{m}^{2}$. 
The smaller intensity ratio (edge to center) obtained from anthracene can be attributed to self-absorption of the emitted fluorescence within the crystal /13-16/. The fluorescence and absorption spectra of anthracene crystals are considerably overlapped; therefore, the fluorescence light directed towards the edges by the crystal wave-guide undergoes considerable self-absorption. The fluorescence spectra of perylene crystals, in contrast, are only slightly overlapped with the absorption spectra /17/. Therefore, less self-absorption of the fluorescence light is expected for perylene crystallites.

\section{c. Fluorescence intensity as a function of crystal alignment.}

As we have mentioned earlier, only the apparent area of the crystal is actually being measured. In order to further substantiate this finding, a medium-size perylene crystal $\left(-1000 \mu \mathrm{m}^{2}\right)$ was deposited on a thin glass wire. This was then rotated along its axis, resulting in variable alignments of the crystal towards the excitation light (recall Fig. 4). Since the excitation light projected through the objective was not polarized, no polarization features should be anticipated for in the fluorescence data obtained.

The crystal orientation effects are depicted in Figures. 5 and 6. Fig. 5 presents the fluorescence image of such wire-suspended crystal. A plane cut through F-F' is also specified. The corresponding intensity profiles obtained for different crystal orientations with respect to the microscope aperture are shown in Figures. 6.

In Fig. 6a, which corresponds to the intensity profile of the abovespecified F-F' cut, three regions of maximal intensity are noted. High emission at the crystal edges is due to the aforementioned wave-guiding effect. The peak at the crystal's central region might be due to structural imperfections (see Figures $2 a$ and 5) through which, similar to the crystal edges, the light escapes.

Figures $6 \mathrm{~b}$ and $6 \mathrm{c}$, which show the intensity cross-sections of the crystal, when rotated at opposite directions, indicate the dependence of the imaged fluorescence on the crystal alignment. Figure $6 \mathrm{~d}$ shows the intensity crosssection of the smallest crystal's facet (see Figure $2 a$ ). In this case only a single peak is observed, indicating that the wave-guiding effect is intrinsically related to the crystal structure.

These findings hence substantiate that, although the fluorescence yield is an inherent property of any PAH crystal, the observed (i.e., detected) emission depends upon the crystallite's alignment towards the objective. 


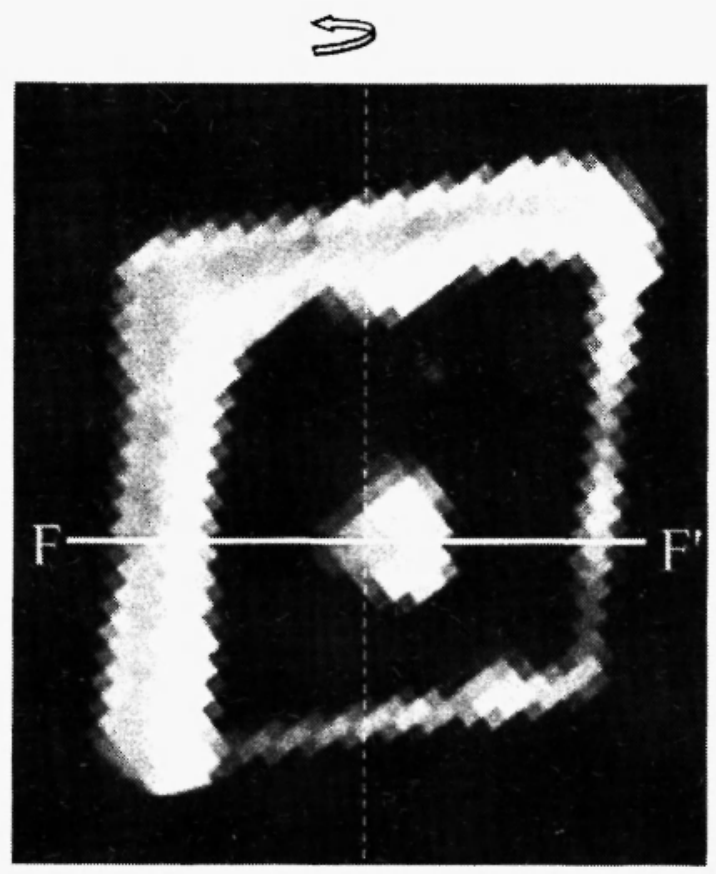

Fig. 5: A specified plane cut through, (F-F') illustrated for a perylene crystal. The arrow indicates the rotation axis.

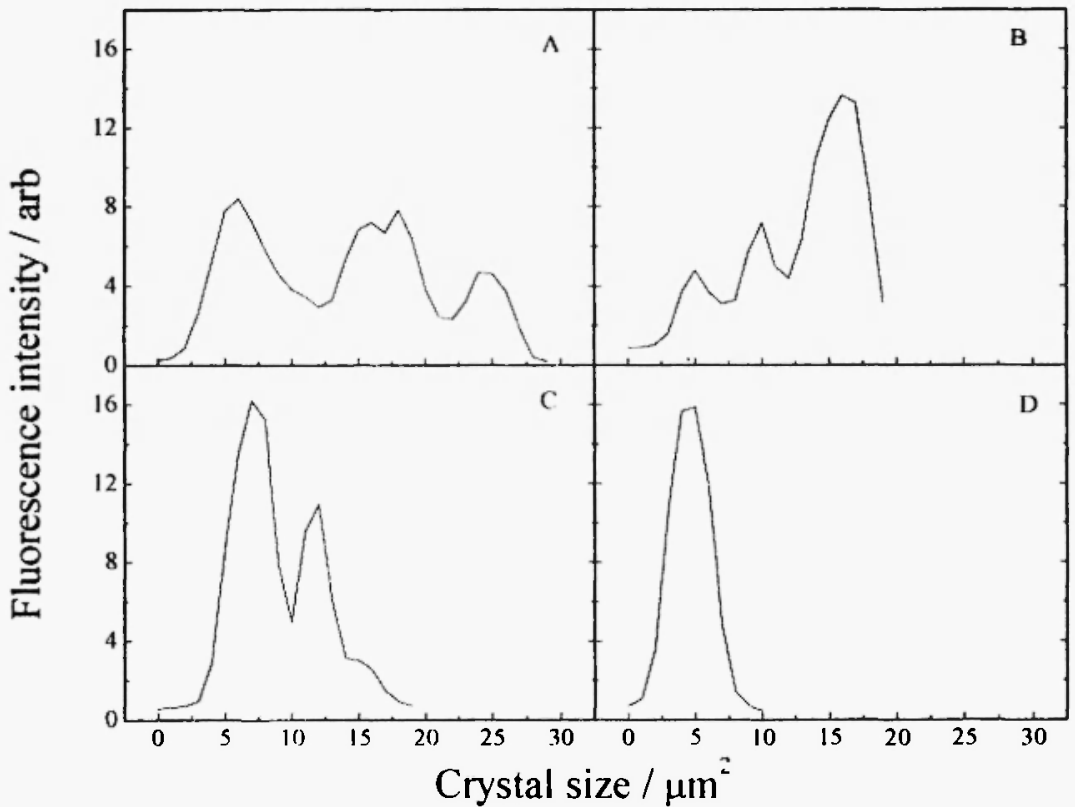

Fig. 6: Fluorescence intensity along F-F' for several crystal alignments. 


\section{Spectral analysis}

The results shown so far have pointed out that characterization of bulk $\mathrm{PAH}$ fluorescence using microscope imaging methods requires a proper sampling and inspection of many individual particles (micro-crystals). Statistically sound observations thus result. This was found to be true for the spectral analysis of the particulate matter as well.

Typical fluorescence spectra obtained from the central and the edge neighborhood of perylene crystals are shown in Figure 7. It is noteworthy that the fluorescence spectra from the central region are in agreement with the results obtained by other research groups $/ 17,18 /$. There, the fluorescence spectra of an $\alpha$-perylene crystallite were represented by a broad structureless band. The latter was considerably red-shifted relative to that from the corresponding solution of perylene.

The two noticeable bands of Figure 7 are probably due to structural defects located at the surface. This is substantiated by the edge-originated

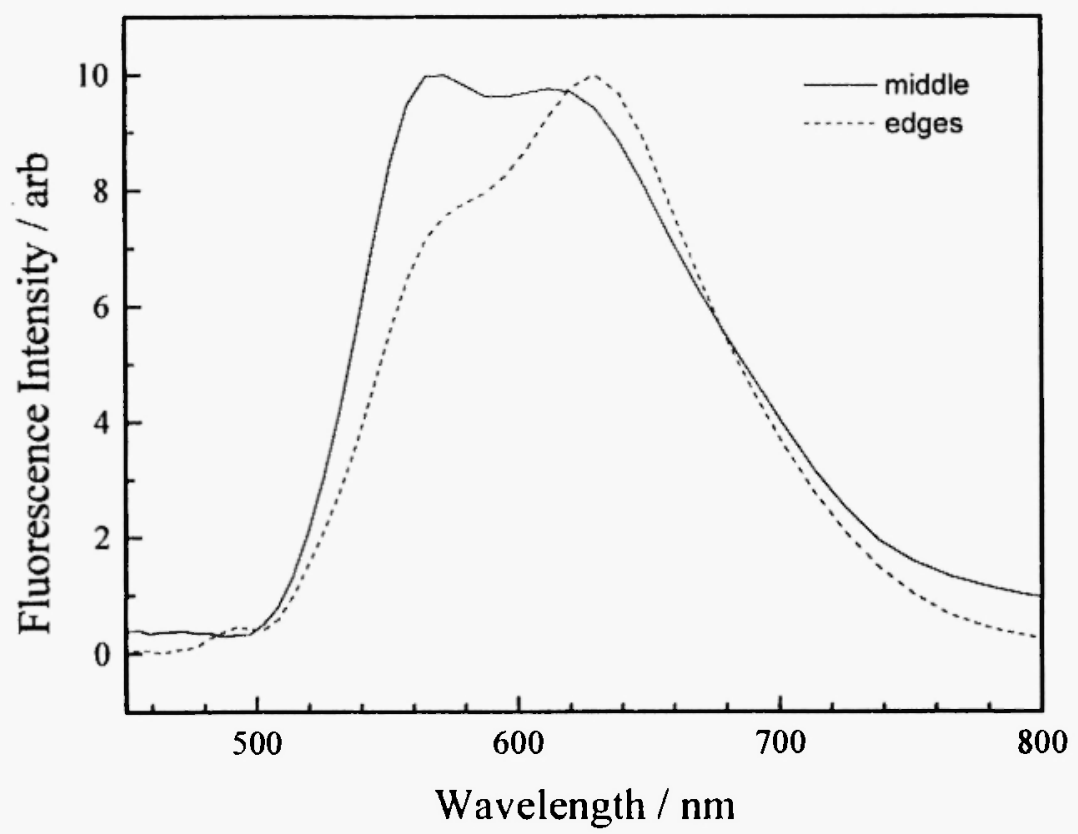

Fig. 7: Fluorescence spectra at the edges and at the central region of a perylene crystal. 
spectra shown. Note that, due to the simple geometric considerations, more surface defects are observed at the edges.

Since the microscope observes a $2 \mathrm{D}$ projection of the crystal, the edgeregion includes all defects on the lateral facets. It appears that the fluorescence spectra due to crystal defects is somewhat red-shifted. Tamai et al. $/ 19 /$ have also noticed this for $\beta$-perylene crystals. It was assigned to the inherently increased defect concentration at their edges, whereby the selftrapped exciton emission takes place.

Another interesting aspect of FTIM is related to emission originating from the non-irradiated regions. Poor identification of PAH materials might then result. Investigation of this issue requires imaging spectroscopy of single crystals and this is the first report of such data.

For this purpose, the middle regions of large crystallites were irradiated through a mechanical aperture positioned in front of the light source. Resulting fluorescence patterns obtained at different locations were then collected. Diffraction induced intensity variations were not observed under our experimental conditions.

A typical spectral analysis of a large (ca. $0.4 \mathrm{~mm}^{2}$ ) perylene crystal is shown in Fig. 8. Due to its large area, it was possible to illuminate selectively a portion of its central region. A rather sharp irradiation pattern was achieved, as is seen in the insert of Fig. 8. The spectral analysis was performed on the illuminated regions as well as on the non-illuminated ones. It is evident that the fluorescence emitted from the non-illuminated regions was considerably red-shifted, possessing a wide band around $650 \mathrm{~nm}$.

Concurrently, we have no clear-cut explanation for this effect, although the red shift is more significant for large crystals than for the small ones. It would indicate that the red-shifting could be also attributed to the longer path through the crystal wave-guide, and not only to the aforementioned surface defects. The fluorescence from the illuminated region seems to resemble the spectra from the central region of smaller crystals. This finding provides independent evidence for the surface layer probing capacity of the system (refer to the fluorescence intensity related features discussed above).

As previously mentioned, perylene crystals undergo non-significant selfabsorption; thus the above findings cannot be entirely attributed to this effect. However, the absorption and emission spectral overlapping in anthracene allows for the self-absorption features to show up. Therefore, in order to investigate this effect, a batch of anthracene crystals was similarly analyzed 


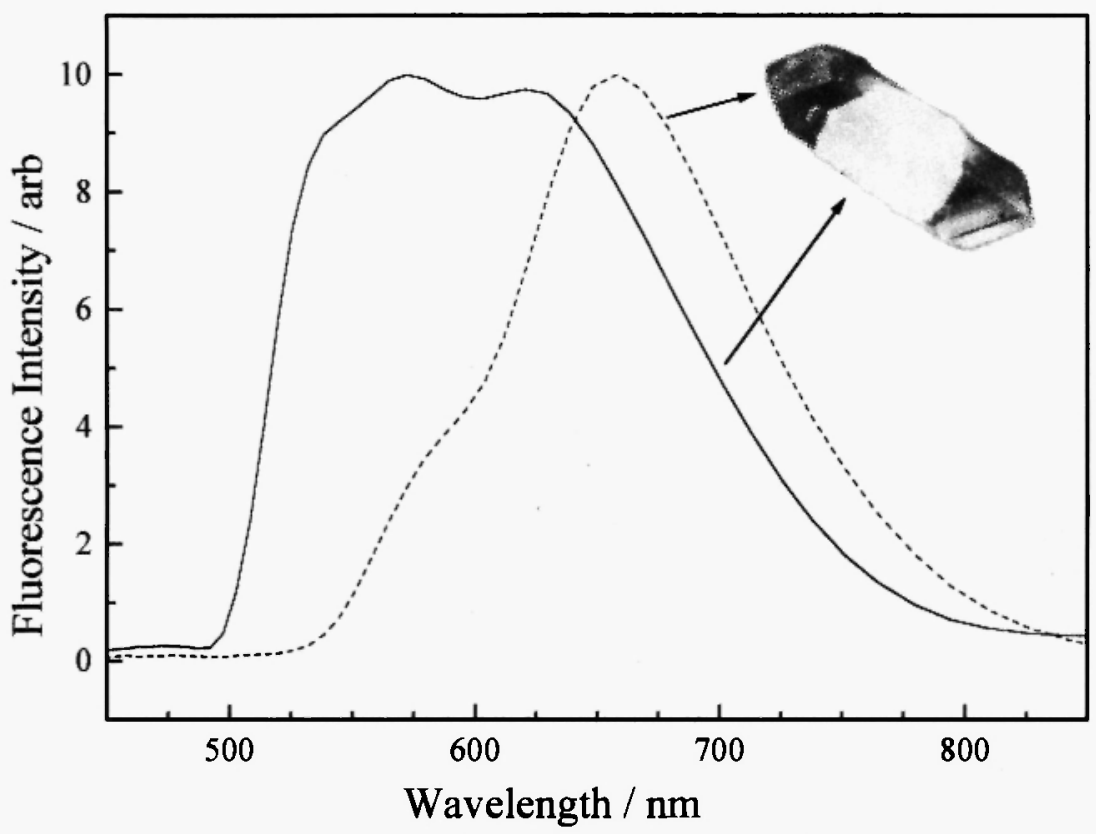

Fig. 8: Fluorescence spectra of a large perylene crystal, measured at a UV illuminated region and at a non-illuminated part. The inset shows the actual fluorescence pattern. illustrating the illuminated and the nonilluminated crystal parts.

and the corresponding spectra for the central and edge locations are shown in Fig. 9. The self-absorption phenomenon within the single crystal is selfevident.

Indeed, the fluorescence spectra at the edges show that the band at 420 $\mathrm{nm}$ is largely reduced. Moreover it is accompanied with an increased band at $440 \mathrm{~nm}$. Such observations /14,15/ are usually attributed to self-absorption of the fluorescence light. Nevertheless, we cannot disregard the possibility that, similar to perylene crystals, additional mechanisms take place.

\section{CONCLUSIONS}

In the present study we have demonstrated some of the problems encountered during direct analysis of particulate material by luminescence methods. Although spectral imaging is a useful tool for the analysis of particulate materials, several quantification aspects have to be considered. 


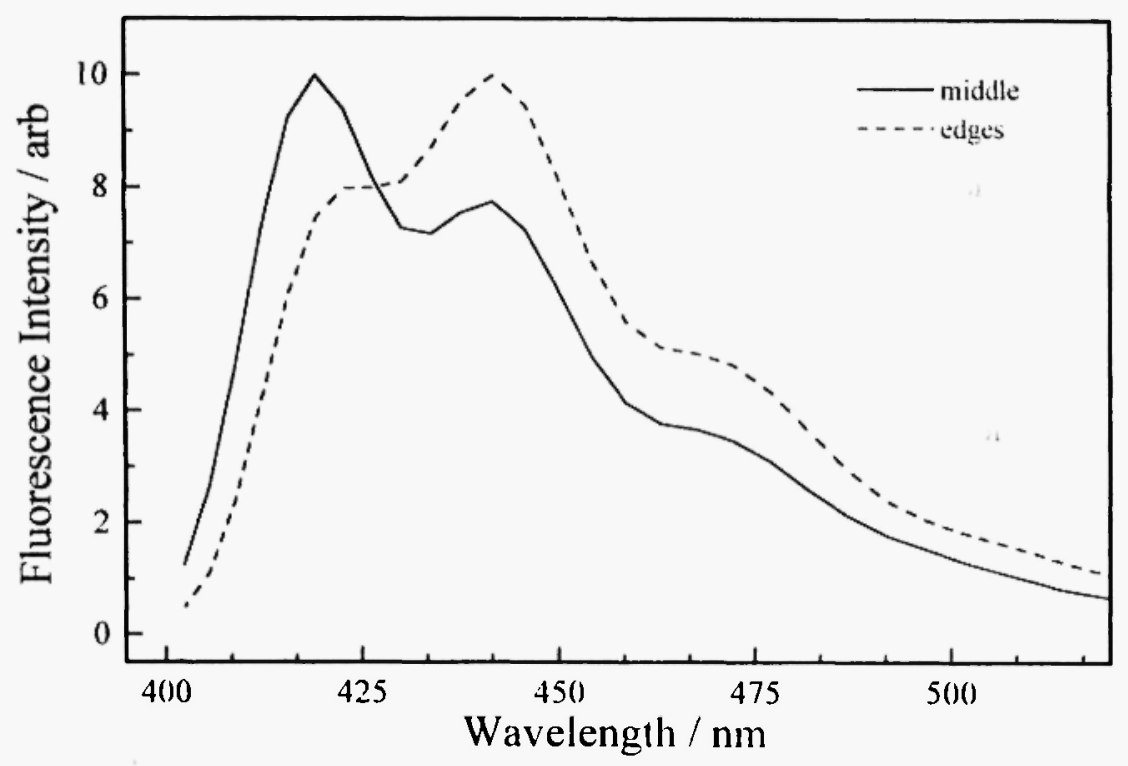

Fig. 9: Fluorescence spectra measured at the central part of an anthracene crystal and at its edges.

These include morphological and temporal effects, which require information on single crystals.

Our results have shown, for example, that the fluorescence of crystalline PAHs is related to specified regions in the crystals. The specific geometrical alignment of the crystals may determine fluorescence intensity and its spectrum. Internal wave-guiding and self-absorption may play a considerable role in material speciation. These effects, too, have to be taken into consideration for a better quantitative analysis.

The variety of spectral features attainable by FTIM qualifies this method for analytical purposes, as well as for detailed studies of luminescence of organic crystallites.

\section{ACKNOWLEDGMENTS}

Collaboration with D. Moshe and N. Horesh of Green Vision Systems Ltd. is much appreciated. This research was supported, in part, by the James Franck Program in Laser Matter Interaction and by the Israel Ministry of the Environment. VVG and VB are grateful for financial support by the Israel Ministry of Absorption, given to new immigrant scientists. 


\section{REFERENCES}

1. T. Vo-Dinh (Ed.) Chemical Analysis of Polycyclic Aromatic Compounds, Wiley, New York, 1990.

2. D.A. Dzombak and R.G. Luthy, Soil Sci, 137, 292 (1984).

3. A.M. Mastral, M. Callen and R. Murillo, Polycyclic Arom. Comp., 9, 37 (1996).

4. D.A. Lane, "Polycyclic aromatic hydrocarbons: atmospheric physics and chemistry"; In Organic Chemistry of the Atmosphere; L.D. Hansen, D.J. Eatough (Eds.); CRC Press: Boca Raton, 1991; Chapter 5.

5. M. Katz and C. Chan, Environ. Sci. Technol, 14, 838 (1980).

6. M.N. Kayalisayadi, L.M. Rubiobarraso and J. Beceiroldan, Liq. Chrom. Rel. Tech., 19, 3135 (1996).

7. W. Chudyk, Environ. Sci. Tech., 23, 504 (1989).

8. J.W. Koehn and G.H. Stanko, Environ. Sci. Tech., 22, 1262 (1988).

9. R. Niessner, U. Panne and H. Schroder, Anal. Chim. Acta, 255, 231 (1991).

10. A.N. Gennandiyev, Y.I. Pikovsky and I.S. Kozin, Polyclic Arom. Comp., 9, 169 (1996).

11. I.S. Kozin, N.H. Gooijer, J. Velthorst and R. Harmsen, J. Environ. Anal. Chem., 61, 285 (1995).

12. C. Sluszny, V. Bulatov and I. Schechter, Anal. Chim. Acta, 367, 1 (1998).

13. E.J. Bowen and P.D. Lawley, Nature, 164, 572 (1949).

14. J.B. Birks and G.T. Wright, Proc. Phys. Soc., 67(B), 657 (1954).

15. J.B. Birks and J.W. Cameron, Proc. Roy. Soc., A249, 297 (1959).

16. J.B. Birks, Mol. Cryst. Liq. Cryst., 28, 117 (1973).

17. J. Tanaka, Bull. Chem. Soc. Japan, 36, 1237 (1963).

18. B. Walter, H. Port and H.C. Wolf, Chem. Phys., 92, 177 (1985).

19. N. Tamai, C.F. Porter and H. Masuhara, Chem. Phys. Lett., 211, 364 (1993). 